\title{
Heavy Quark Fragmentation to Polarised Quarkonium
}

\author{
Adam F. Falk ${ }^{\dagger}$, Michael Luke ${ }^{b}$, Martin J. Savage $^{b} \S \star$ and Mark B. Wise ${ }^{c}$ \\ a) Stanford Linear Accelerator Center, Stanford CA 94309 \\ b) Department of Physics, University of California at San Diego, \\ 9500 Gilman Drive, La Jolla CA 92093 \\ c) California Institute of Technology, Pasadena CA 91125
}

\begin{abstract}
We calculate the polarisation of $\psi$ 's and $\Upsilon$ 's produced by the fragmentation of heavy quarks. We find that fragmentation to transversely aligned quarkonium is slightly enhanced relative to longitudinally polarised. This net alignment corresponds to a $\sim 5 \%$ asymmetry in the angular distribution of leptons produced in the subsequent decay of the quarkonium. We point out that the leading gluon contribution to $\psi$ and $\Upsilon$ production arises from the evolution of the charm quark fragmentation function, rather than from direct gluon fragmentation.
\end{abstract}

UCSD/PTH 93-06, CALT-68-1864, SLAC-PUB-6175

hep-ph/9305260

May 1993

$\dagger$ Address after 10/1/93: Dept. of Physics, UC San Diego, La Jolla, CA 92093

$\ddagger$ Address after 9/1/93: Dept. of Physics, University of Toronto, Toronto, Ontario, Canada M5S 1A7

$\S$ Address after 9/1/93: Dept. of Physics, Carnegie Mellon University, Pittsburgh, PA 15213

* SSC Fellow 
Quarkonium production in high energy processes is dominated by the fragmentation of heavy quarks and gluons [1]- [4]. For example, in $Z^{0}$ decay the short distance process $Z^{0} \rightarrow \psi g g$ is suppressed relative to the fragmentation process $Z^{0} \rightarrow \psi c \bar{c}$ by a factor of $m_{c}^{2} / m_{Z}^{2}$, corresponding to the small overlap of the $\psi$ wavefunction with a $c \bar{c}$ pair that has relative momentum of order $m_{Z}$.

Recently it has been shown by Braaten and Yuan [3] and Braaten, Cheung and Yuan [4] that the process independent fragmentation functions for quarkonium production in high energy processes are calculable. For example, in $Z^{0}$ decay to a $\psi$ of energy $E \gg m_{\psi}$, the differential decay rate may be written in the factorised form

$$
d \Gamma\left(Z^{0} \rightarrow \psi(E)+X\right)=\sum_{i} \int_{0}^{1} d z d \widehat{\Gamma}\left(Z^{0} \rightarrow i(E / z)+X, \mu\right) D_{i \rightarrow \psi}(z, \mu) .
$$

Here $d \widehat{\Gamma}\left(Z^{0} \rightarrow i(E / z)+X, \mu\right)$ is the differential decay rate for a $Z^{0}$ to decay to an on-shell parton of type $i$, while the fragmentation function $D_{i \rightarrow \psi}(z, \mu)$ gives the probability for the parton to split into a $\psi$ with momentum fraction $z$ and another parton with momentum fraction $1-z$. The advantage of this factorised form is that the fragmentation function is independent of the production process. QCD corrections of the form $\log \left(m_{Z} / m_{c}\right)$ necessitate the introduction of a factorisation scale $\mu$; large logarithms of $\mu / m_{c}$ in $D_{i \rightarrow \psi}(z, \mu)$ may be summed using the Altarelli-Parisi equations [5] [6].

The fragmentation functions $D_{c \rightarrow \psi}(z, \mu)$ and $D_{b \rightarrow \Upsilon}(z, \mu)$ were calculated in [4], explicitly summing over quarkonium polarisations. However, the fragmentation functions for transverse and longitudinally polarised quarkonium are individually calculable and are interesting in their own right. In this work we compute the fragmentation function for production of transversely polarised $\psi$ 's from $c$ quarks and $\Upsilon$ 's from $b$ quarks.

The Feynman diagrams responsible for the fragmentation of a heavy quark into quarkonium are shown in fig. 1, where the black circle represents some quark-antiquark production process. For definiteness we consider $Z^{0}$ decay to $\psi c \bar{c}$; however the fragmentation function we will derive is independent of the production process and is trivially extended to $b$ fragmentation to $\Upsilon$. Factorisation is only manifest in $\bar{q} \cdot A=0$ gauge (where $\bar{q}$ is the antiquark four-momentum), in which the diagram in fig. 1(b) vanishes to leading order in $m_{c} / E$. Following the manipulations in Eqs. (9)-(11) of Ref. [4, we write the decay rate $\Gamma\left(Z^{0} \rightarrow \psi(\epsilon) c \bar{c}\right)$, averaging over initial spins and summing over colours, as

$$
\begin{aligned}
\Gamma\left(Z^{0} \rightarrow \psi(\epsilon) c \bar{c}\right)=\frac{1}{2 m_{Z}} & \int[d q][d \bar{q}](2 \pi)^{4} \delta^{(4)}\left(p_{Z}-\bar{q}-q\right) \\
& \times \int_{0}^{1} \frac{d z}{8 \pi} \int_{0}^{\infty} \frac{d s}{2 \pi} \theta\left(s-\frac{4 m_{c}^{2}}{z}-\frac{m_{c}^{2}}{1-z}\right)|A(\epsilon)|^{2},
\end{aligned}
$$


where $A(\epsilon)$ is the amplitude to produce a $\psi$ with polarisation $\epsilon, p_{Z}$ is the $Z^{0}$ fourmomentum, $s=q^{2}$ is the invariant mass of the original $c$ quark, $[d q] \equiv d^{3} q /\left(16 \pi^{3} q_{0}\right)$, and $z$ is the longitudinal momentum fraction of the $\psi$. Choosing a frame such that $q=\left(q^{0}, 0,0, q^{3}\right)$, we have $z=\left(p_{0}+p_{3}\right) /\left(q_{0}+q_{3}\right)$, where $p$ is the $\psi$ four-momentum.

Squaring the result from fig. 1 and using the identity

$$
\sum_{T} \epsilon_{i}^{T} \epsilon_{j}^{* T}=\delta_{i j}-\frac{p_{i} p_{j}}{\vec{p}^{2}}
$$

to sum over transverse $\psi$ polarisations, we find

$$
\sum_{T}|A(\epsilon)|^{2}=\frac{1}{\left(s-m_{c}^{2}\right)^{4}} D^{T}(s, z)\left(-g^{\alpha \beta}+\frac{p_{Z}^{\alpha} p_{Z}^{\beta}}{m_{Z}^{2}}\right) \operatorname{tr}\left(\Gamma_{\alpha} \not \bar{q} \Gamma_{\beta} \not\right)
$$

where

$$
\begin{array}{r}
D^{T}(s, z)=\frac{256}{81} \pi^{2} \alpha_{s}^{2} f_{\psi}^{2}\left[8 m_{c}^{2} s\left(z-2+\frac{2}{z}\right)-8 m_{c}^{4}\left(z+4-\frac{6}{z}+\frac{8}{z^{2}}\right)\right. \\
\left.+\frac{16 m_{c}^{2}\left(s-m_{c}^{2}\right) z^{2}}{2-z}+\frac{8\left(s-m_{c}^{2}\right)^{2} z^{2}(1-z)}{(2-z)^{2}}\right]
\end{array}
$$

We have dropped terms suppressed by $m_{c} / E$ in our result. $f_{\psi}$ is the $\psi$ decay constant, defined by

$$
\left\langle 0\left|\bar{c} \gamma^{\mu} c\right| \psi(p, \epsilon)\right\rangle=f_{\psi} m_{\psi} \epsilon^{\mu}
$$

This is related to the nonrelativistic radial wavefunction at the origin, $R_{\psi}(0)$, by $f_{\psi}=$ $\sqrt{3 / \pi m_{\psi}} R_{\psi}(0)$. Numerically, from the $\psi$ leptonic width, $f_{\psi} \approx 410 \mathrm{MeV}$. The total decay rate to transversely aligned $\psi$ 's may now be written in the factorised form

$$
\begin{aligned}
\Gamma\left(Z^{0} \rightarrow \psi^{T} c \bar{c}\right)= & \frac{1}{16 \pi^{2}} \Gamma\left(Z^{0} \rightarrow c \bar{c}\right) \\
& \quad \times \int_{0}^{1} d z \int_{0}^{\infty} d s \theta\left(s-\frac{4 m_{c}^{2}}{z}-\frac{m_{c}^{2}}{1-z}\right) \frac{1}{\left(s-m_{c}^{2}\right)^{4}} D^{T}(s, z),
\end{aligned}
$$

where the decay rate to free quarks is

$$
\begin{aligned}
\Gamma\left(Z^{0} \rightarrow c \bar{c}\right)=\frac{1}{2 m_{Z}} & \int[d q][d \bar{q}](2 \pi)^{4} \delta^{(4)}\left(p_{Z}-q-\bar{q}\right) \\
& \times\left(-g^{\alpha \beta}+\frac{p_{Z}^{\alpha} p_{Z}^{\beta}}{m_{Z}^{2}}\right) \operatorname{tr}\left(\Gamma_{\alpha} \bar{\phi} \Gamma_{\beta} \phi\right) .
\end{aligned}
$$


Performing the integral over $s$ in (7), we find the expression for the fragmentation function to transversely aligned $\psi$ 's:

$$
\begin{aligned}
D_{c \rightarrow \psi}^{T}\left(z, \mu=3 m_{c}\right)=\frac{16}{81 m_{c}^{2}} & \alpha_{s}\left(3 m_{c}\right)^{2} f_{\psi}^{2} \\
& \times \frac{2}{3} \frac{z(1-z)^{2}}{(2-z)^{6}}\left(16-32 z+76 z^{2}-36 z^{3}+6 z^{4}\right) .
\end{aligned}
$$

Adding the longitudinal polarisation, we recover the total fragmentation function $D_{c \rightarrow \psi}^{T+L}(z)$ given in [4],

$$
\begin{aligned}
D_{c \rightarrow \psi}^{T+L}\left(z, \mu=3 m_{c}\right)=\frac{16}{81 m_{c}^{2}} & \alpha_{s}\left(3 m_{c}\right)^{2} f_{\psi}^{2} \\
& \times \frac{z(1-z)^{2}}{(2-z)^{6}}\left(16-32 z+72 z^{2}-32 z^{3}+5 z^{4}\right) .
\end{aligned}
$$

In experiments performed at a hadron collider, the gluon fragmentation function also plays an important role. The Altarelli-Parisi evolution of $D_{c \rightarrow \psi}^{T(T+L)}(z, \mu)$ from the scale $\mu=3 m_{c}$ to a high energy scale $\mu=M$ (such as $M=m_{Z}$ ) will induce a gluon fragmentation function $D_{g \rightarrow \psi}^{T(T+L)}(z, M)$ via the gluon splitting function $P_{g \rightarrow c \bar{c}}(z)$. That is, a high energy gluon may split into a quark-antiquark pair of lower energy, either of which may then fragment to quarkonium. In the leading logarithmic approximation this effect, of order $\alpha_{s}^{3} \ln \left(M / m_{c}\right)$, dominates over the direct contribution to gluon fragmentation computed in [3], which is of order $\alpha_{s}^{3}$ without the $\ln \left(M / m_{c}\right)$ enhancement. ${ }^{\dagger}$

It is equally straightforward to evolve the quark fragmentation functions (9) and (10) to high energies using the Altarelli-Parisi equations and the quark splitting functions $P_{c \rightarrow c g}(z)$ and $P_{c \rightarrow g c}(z)=P_{c \rightarrow c g}(1-z)$. Because $\int_{0}^{1} d z P_{c \rightarrow c g}(z)=0$, this evolution softens the $z$ distributions of $D_{c \rightarrow \psi}^{T}(z, \mu)$ and $D_{c \rightarrow \psi}^{T+L}(z, \mu)$ but leaves their integrals $\int_{0}^{1} d z D_{c \rightarrow \psi}^{T(T+L)}(z, \mu)$ unchanged [4].

We now define $\zeta$ to be the ratio of transverse to total fragmentation probabilities,

$$
\zeta=\frac{\int_{0}^{1} d z D_{c \rightarrow \psi}^{T}(z, \mu)}{\int_{0}^{1} d z D_{c \rightarrow \psi}^{T+L}(z, \mu)} .
$$

As discussed in the previous paragraph, $\zeta$ is independent of $\mu$. Evaluating (11) at $\mu=3 m_{c}$, we find $\zeta=0.69$, to be compared with $\zeta=\frac{2}{3}$ for production of unaligned $\psi$ 's. Therefore

$\dagger$ This applies only to $\psi$ and $\Upsilon$ production; gluon fragmentation to $\eta_{c}$ 's and $\eta_{b}$ 's is of order $\alpha_{s}^{2}$, and so the direct gluon fragmentation calculated in [3] dominates. 
a small excess of transversely aligned $\psi$ 's will be produced. Since the dependence on $f_{\psi}$ and $m_{c}$ drops out of (11), the ratio $\zeta$ is the same for $\psi$ 's and $\Upsilon$ 's. In leading logarithmic approximation, the corresponding ratio for gluon fragmentation to $\psi$ 's has the same value and is $\mu$ independent, since the gluon fragmentation functions are induced only by quark fragmentation. Hence, at a hadron collider, where $\psi$ 's are produced both by quark and gluon fragmentation, the fraction of transversely aligned $\psi$ 's is also $\zeta=0.69$.

The asymmetry $\zeta$ is measurable through the angular distribution of the leptons in the decay $\psi \rightarrow \ell^{+} \ell^{-}$. Defining $\theta$ to be the angle between the alignment axis and the lepton momentum, the angular distribution $d \Gamma / d \theta$ in the $\psi$ rest frame has the form

$$
\frac{d \Gamma\left(\psi \rightarrow \ell^{+} \ell^{-}\right)}{d \theta} \propto 1+\alpha \cos ^{2} \theta
$$

where

$$
\alpha=\frac{3 \zeta-2}{2-\zeta}
$$

$\zeta=0.69$ corresponds to $\alpha=0.053$, about a $5 \%$ asymmetry.

It is useful to compare this with the polarisation of $\psi$ 's produced from nonleptonic $b$ decay. Assuming the four-quark amplitude factorises (which should give a reasonable estimate, as the $\psi$ is a compact object on the hadronic scale $\Lambda_{Q C D}$ ), a straightforward calculation of the decay $b \rightarrow \psi s \rightarrow \ell^{+} \ell^{-} s$ gives

$$
\alpha=-\frac{m_{b}^{2}-m_{\psi}^{2}}{m_{b}^{2}+m_{\psi}^{2}} \approx-0.46
$$

This is in nice agreement with a recent CLEO inclusive measurement, which finds [7] (see also [8])

$$
\alpha=-0.44 \pm 0.16
$$

(where we have added the systematic and statistical errors linearly). Since the inclusive branching ratio for $b \rightarrow \psi X$ is $(1.02 \pm 0.05 \pm 0.09) \%$ [7], this decay may provide a significant background to $\psi$ production by fragmentation at high energy colliders. There is no comparable decay process to compete with $b$ quark fragmentation for $\Upsilon$ production; therefore this mode should allow a cleaner measurement of $\zeta$.

We note that $\psi$ ( or $\Upsilon$ ) production can also arise from the fragmentation into $\mathrm{P}$-wave quarkonium which subsequently decays electromagnetically to $\psi$ (or $\Upsilon$ ), as mentioned in [4]. For weakly bound nonrelativistic quarkonium, the fragmentation into P-wave states 
is suppressed; however, in the charmonium system this may be an important additional source of $\psi$ 's.

In summary, we have calculated the leading contribution to the transverse fragmentation function for quarkonium production from heavy quarks. We find that the $\psi$ and $\Upsilon$ are essentially unaligned, with only a $\sim 5 \%$ angular anisotropy in the lepton distribution produced in their electromagnetic decays. We also point out that the dominant contribution to gluon fragmentation into $\psi$ or $\Upsilon$ in high energy processes arises from Altarelli-Parisi evolution of heavy quark fragmentation functions, rather than from direct gluon fragmentation.

This work was supported in part by the Department of Energy under contracts DE-AC03-76SF00515 (SLAC), DE-FG03-90ER40546 (UC San Diego) and DE-FG0392ER40701 (Caltech). MJS acknowledges the support of a Superconducting Supercollider National Fellowship from the Texas National Research Laboratory Commission under grant FCFY9219. 


\section{References}

[1] J.H. Kühn and H. Schneider, Phys. Rev. D24 (1981) 2996

[2] V. Barger, K. Cheung and W.Y. Keung, Phys. Rev. D41 (1990) 1541

[3] E. Braaten and T.C. Yuan, NUHEP-TH-92-23, UCD-92-25 (1992)

[4] E. Braaten, K. Cheung and T.C. Yuan, NUHEP-TH-93-2,UCD-93-1 (1993)

[5] G. Altarelli and G. Parisi, Nucl. Phys. B126 (1977) 298

[6] R. B. Field, Applications of Perturbative QCD, Addison Wesley (1989)

[7] The CLEO Collaboration (A. Bean et al.), "Decay Rates and Polarization in Inclusive and Exclusive $B \rightarrow \psi$ Decays", Talk presented at the 1992 International Conference on High Energy Physics, Dallas, Texas

[8] H. Schröder, "ARGUS Results on $B$ Decays via $b \rightarrow c$ Transitions", published in Proceedings of the 25th International Conference on High Energy Physics, K. K. Phua and Y. Yamaguchi, eds., World Scientific (1991) 


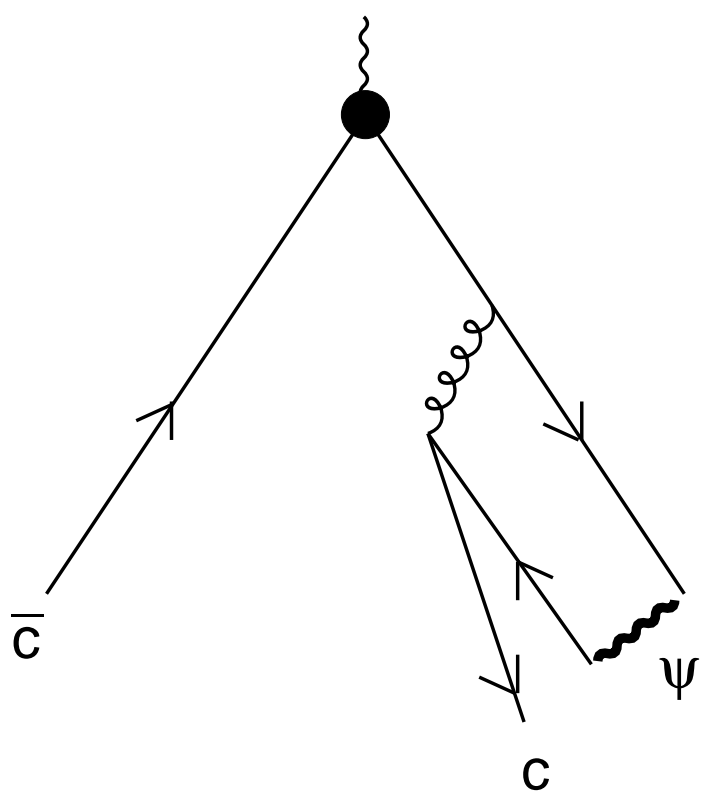

(a)

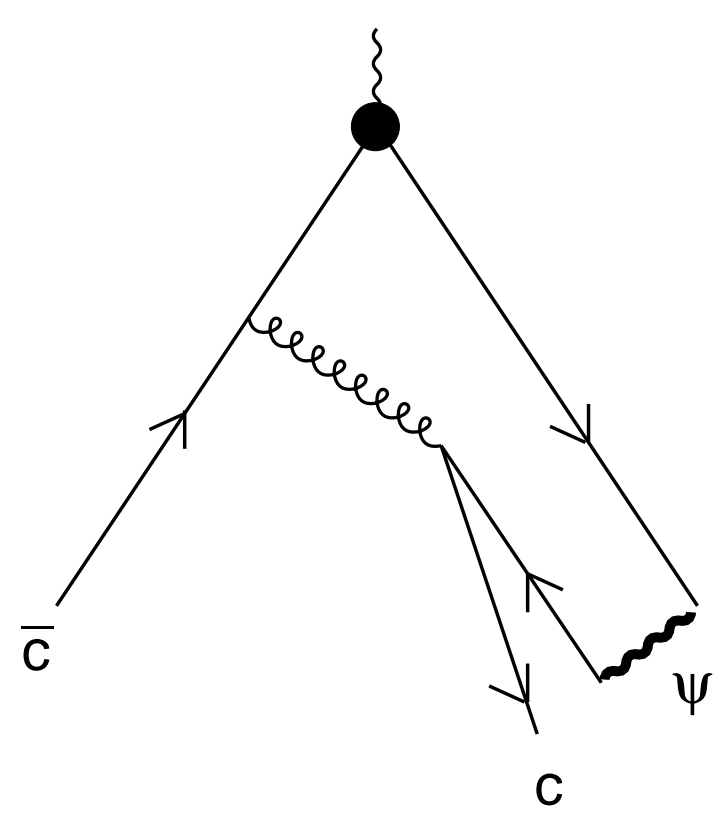

(b)

Fig. 1. Feynman diagrams responsible for the fragmentation $c \rightarrow \psi c$. 